\title{
Quantitative phosphoproteomic analysis of prion-infected neuronal cells
}

Wibke Wagner ${ }^{1}$, Paul Ajuh², Johannes Löwer ${ }^{1}$, Silja Wessler ${ }^{1,3^{*}}$

\begin{abstract}
Prion diseases or transmissible spongiform encephalopathies (TSEs) are fatal diseases associated with the conversion of the cellular prion protein $\left(\operatorname{PrP}^{\mathrm{C}}\right)$ to the abnormal prion protein $\left(\mathrm{PrP}^{\mathrm{SC}}\right)$. Since the molecular mechanisms in pathogenesis are widely unclear, we analyzed the global phospho-proteome and detected a differential pattern of tyrosine- and threonine phosphorylated proteins in PrPSC-replicating and pentosan polysulfate (PPS)-rescued N2a cells in two-dimensional gel electrophoresis. To quantify phosphorylated proteins, we performed a SILAC (stable isotope labeling by amino acids in cell culture) analysis and identified 105 proteins, which showed a regulated phosphorylation upon PrPSc infection. Among those proteins, we validated the dephosphorylation of stathmin and $\mathrm{Cdc2}$ and the induced phosphorylation of cofilin in PrPSc-infected N2a cells in Western blot analyses. Our analysis showed for the first time a differentially regulated phospho-proteome in $\mathrm{PrP}^{\mathrm{Sc}}$ infection, which could contribute to the establishment of novel protein markers and to the development of novel therapeutic intervention strategies in targeting prion-associated disease.
\end{abstract}

\section{Findings}

Transmissible spongiform encephalopathies (TSEs) are fatal neurodegenerative diseases occurring in many different host species including humans, which develop e.g. Creutzfeld Jacob disease (sCJD) [1]. The development of TSEs is associated with the self-propagating conversion of the normal host cellular prion protein $\left(\operatorname{PrP}^{\mathrm{C}}\right)$ into the abnormal protease-resistant isoform $\left(\mathrm{PrP}^{\mathrm{Sc}}\right.$ or $\left.\operatorname{PrP}^{\mathrm{res}}\right)$ in an autocatalytic manner [2]. $\mathrm{PrP}^{\mathrm{Sc}}$ plays a key role as an infectious agent in certain degenerative diseases of the central nervous system [3].

The cellular functions of $\mathrm{PrP}^{\mathrm{C}}$ and $\mathrm{PrP}^{\mathrm{Sc}}$ still remain enigmatic. The cellular prion protein can be variably glycosylated at two N-glycosylation sites and is C-terminally attached to the cell surface by a glycosyl phosphatidylinositol (GPI) anchor. GPI-anchored proteins are found in lipid rafts, highly cholesterol- and glycolipid-enriched membrane domains associated with a large number of signaling molecules such as G-protein-coupled receptors and protein kinases suggesting that signaling transduction pathways might play a role in TSEs [4]. Hence, previous publications described a functional role of $\operatorname{Pr} \mathrm{P}^{\mathrm{C}}$ as a signaling molecule with major findings indicating

\footnotetext{
* Correspondence: silja.wessler@sbg.ac.at

'Paul Ehrlich Institute, Paul Ehrlich-Straße 51-59, D-63225 Langen, Germany
} Full list of author information is available at the end of the article that $\operatorname{PrP}^{\mathrm{C}}$ interacts with and activates Src family kinases [5-7]. Increased levels of active Src kinases in scrapieinfected cells then led to the activation of downstream signal transduction pathways [8]. Recently, activation of the JAK-STAT signaling pathway in astrocytes of scrapie-infected brains was observed underlining that signal transduction pathways may play pivotal roles in prion pathogenesis [9]. Interestingly, it was demonstrated that inhibition of the non-receptor tyrosine kinase $\mathrm{c}-\mathrm{Abl}$ strongly activates the lysosomal degradation of $\operatorname{Pr} \mathrm{P}^{\mathrm{Sc}}$ [10]. These data indicate that specific interference with cellular signaling pathways could represent a novel strategy in treatment of TSEs.

We have performed a quantitative analysis of the phospho-proteome to obtain a global insight into deregulated signal transduction pathways in scrapie-infected neuronal cells. We analyzed tyrosine- and threonine-phosphorylated proteins in the murine neuroblastoma cell line N2a58/22L, which were infected with the $\operatorname{PrP}^{\mathrm{Sc}}$ strain 22L [11]. We have treated $\mathrm{N} 2 \mathrm{a} 58 / 22 \mathrm{~L}$ cells with pentosan polysulfate (PPS), a known inhibitor of $22 \mathrm{~L} \operatorname{PrP}^{\mathrm{Sc}}$ replication in $\mathrm{N} 2 \mathrm{a}$ cells [12], resulting in the $\mathrm{PrP}^{\mathrm{Sc}}$-rescued cell line N2a58\# which served as an uninfected control. Successful rescue from $\mathrm{PrP}^{\mathrm{Sc}}$ was demonstrated in the colony assay as reflected by the absence of proteinase $\mathrm{K}$ (PK)-resistant $\mathrm{PrP}^{\mathrm{Sc}}$ in N2a58\# cells after PPS treatment (Figure 1A). 

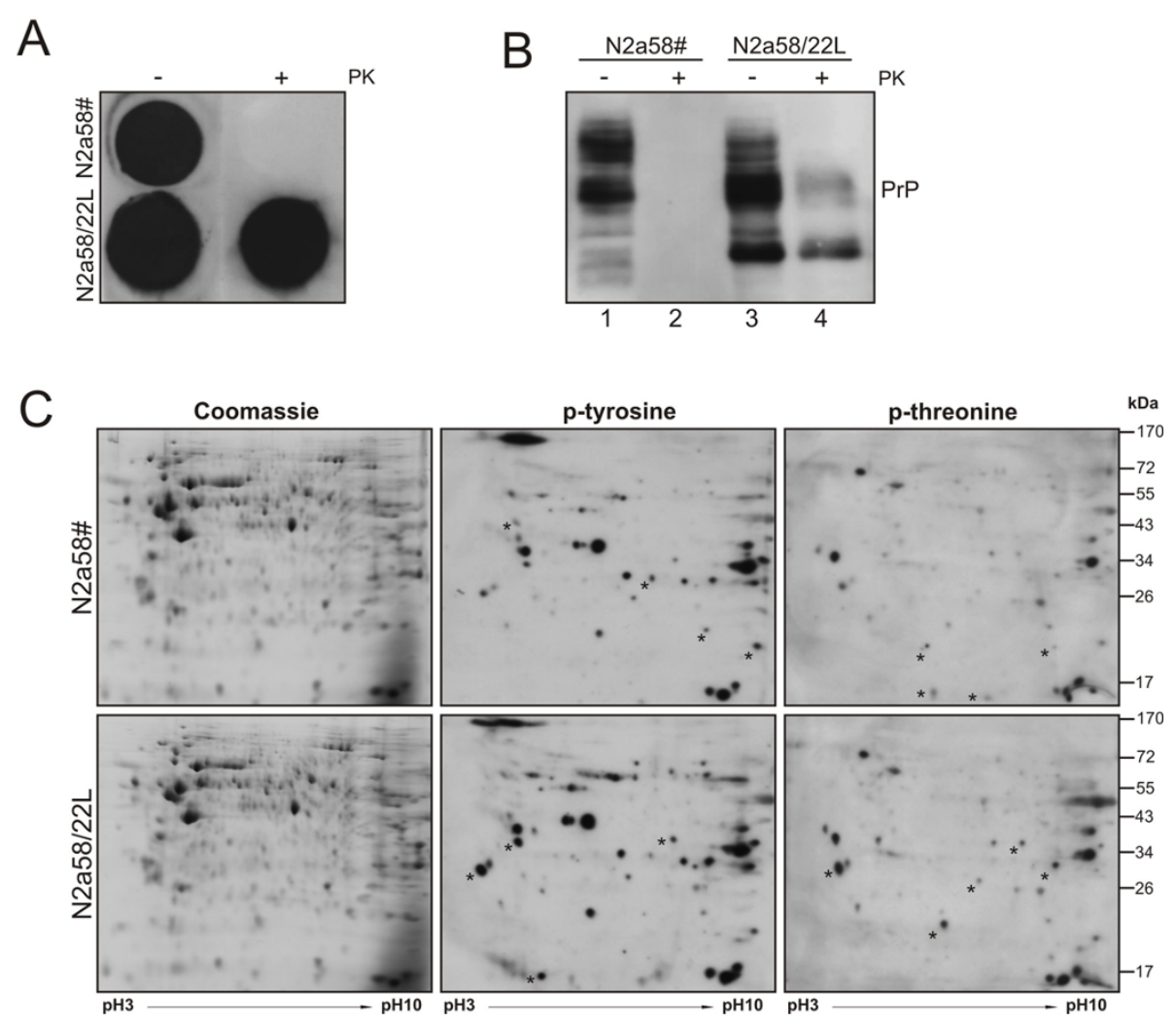

Figure 1 Differentially phosphorylated proteins in $\mathrm{PrP}^{\mathrm{Sc}}$-positive and -negative N2a cells. (A) PrPres-positive N2a58/22L cells were treated with pentosan polysulfate (PPS) to obtain PrPres-negative N2a58\# cells. Successful PPS treatment was validated in a colony assay. Cells were grown to confluence on cover slips and directly lysed on nitrocellulose. Where indicated $20 \mu \mathrm{g} / \mathrm{ml}$ proteinase K (PK) was added followed by the detection of PrP expression using the $6 \mathrm{H} 4$ monoclonal antibody. In non-treated cells (-), PrP was detected in both, cured and infected N2a cells. Upon digestion with PK (+), PrPres was only observed in N2a58/22L cells. (B) Equal amounts of protein lysates were incubated with $20 \mu \mathrm{gg} / \mathrm{ml}$ PK or left untreated. PrP was detected with the $8 \mathrm{H} 4$ monoclonal antibody showing the typical migration pattern of PrP and PrPres in infected and PPS-treated N2a58\# cells. In parallel, lysates were incubated with PK to visualize PK-resistant PrPres in N2a58/22L. (C) 150 $\mu \mathrm{g}$ of N2a58\# or prioninfected N2a58/22L cell lysates were separated by two-dimensional gel electrophoresis followed either by Coomassie staining or immunoblotting for detection of tyrosine- and threonine-phosphorylated proteins. Black asterisks indicate changed intensities of protein phosphorylation.

$\mathrm{PrP}^{\mathrm{Sc}}$ replication and the effect of PPS-treatment were further studied in an immunoblot. After PK digestion, $\mathrm{PrP}^{\mathrm{Sc}}$ replication was only observed in $\mathrm{N} 2 \mathrm{a} 58 / 22 \mathrm{~L}$ cells (Figure 1B, lanes 2 and 4). Compared to 22L-infected N2a58/22L cells, PPS-treated N2a58\# cells showed a different glycosylation profile as expected for $\operatorname{PrP}^{C}$ [13-15]. The glycosylation pattern of $\mathrm{PrP}^{\mathrm{C}}$ in N2a58\# cells displayed high amounts of di- and mono-glycosylated $\operatorname{Pr} \mathrm{P}^{\mathrm{C}}$, whereas in N2a58/22L cells predominantly mono- and non-glycosylated $\operatorname{PrP}^{\mathrm{Sc}}$ was detected (Figure 1B, lanes 1 and 3). Altogether, PPS treatment of N2a58/22L cells successfully abolished $\mathrm{PrP}^{\mathrm{Sc}}$ formation in N2a58\# cells, which served as a non-infected control cell line in our study.

To analyze differentially phosphorylated proteins in N2a58/22L cells in comparison to N2a58\# cells, we separated equal protein amounts by two-dimensional gel electrophoresis. Gels were stained with Coomassie Blue to demonstrate equal protein amounts in N2a58/22L and N2a58\# cells (Figure 1C, left panels). In parallel, gels were blotted onto membranes and incubated with phospho-specific antibodies to detect tyrosine(Figure 1C, middle panels) or threonine-phosphorylated proteins (Figure $1 \mathrm{C}$, right panels). Interestingly, considerable differences in phosphorylation patterns were observed (Figure 1C, asterisks), while other phosphorylated proteins were not changed in N2a58/22L and N2a58\# cells (Figure 1C). These data imply differentially regulated phosphoproteins in response to $22 \mathrm{~L}$ infection of neuronal cells.

Generally, global detection of phosphorylated proteins is still challenging, as antisera often recognize phosphorylated residues dependent on the surrounding sequence. For a general detection of proteins post-translationally phosphorylated at those sites, we performed a SILAC analysis allowing the identification and relative quantification of differential phosphoprotein regulation. Therefore, N2a58\# cells were grown in light isotope containing and N2a58/22L cells in heavy isotope containing 
medium. Equal amounts of protein lysates were mixed, separated by gel electrophoresis, trypsinized and followed by enrichment of phosphoproteins, which were then analyzed by mass spectrometry. We identified 109 different phosphoproteins of which 105 were also quantified (Tables 1 and 2). We observed 75 proteins with a ratio of identified peptides in N2a58/22L versus N2a58\# cells ranging from 0.46 to 0.99 (Table 1). Conversely, 30 phosphoproteins showed a ratio between 1.01 and 1.79 (Table 2). We defined proteins exhibiting a ratio $<0.70$ as dephosphorylated proteins and proteins with ratios between 0.70 and 1.40 as proteins, whose phosphorylation was not altered in $22 \mathrm{~L}$-infected N2a58/22L cells. Ratios $>1.40$ were considered as proteins whose phosphorylation increased upon Scrapie infection.

Among quantified phosphoproteins, we then considered specific phosphosites in selected target proteins, such as Cdc2, stathmin, and cofilin as analyzed by massspectrometry (Table 3). An increase of cofilin ${ }^{\mathrm{S} 3}$ phosphorylation in N2a58/22L cells was suggested by a ratio 1.63 , while the amount of the two tyrosine phosphorylation sites (Y15, Y160) in Cdc2 were decreased upon 22L infection. Stathmin phosphopeptides containing serine 38 were increased, whereas the amount of stathmin phosphopeptides harboring serine 25 in N2a58/22L cells was significantly lower (Table 3 ).

To validate the results obtained in the SILAC phosphoproteomic analysis we performed Western blots for cofilin 1, Cdc2, and stathmin using antibodies for the detection of specific phosphosites. As predicted by the SILAC analysis, cofilin 1 phosphorylation was significantly induced in Scrapie-infected N2a58/22L cells compared to PPS-treated N2a58\# cells (Figure 2, left panels). Cofilin represents a potent regulator of the actin filaments, which is controlled by phosphorylation of serine 3 mediated through the LIM-kinase 1 (LIMK-1) in vitro and in vivo [16]. These data support previous studies indicating a direct interaction of $\mathrm{PrP}^{\mathrm{Sc}}$ with cofilin [17]. Together with our finding that phosphorylation of cofilin is induced in $\mathrm{PrP}^{\mathrm{Sc}}$-infected neuronal cells; the results indicate a significant role for the protein in neurodegeneration processes. Stathmin acts as an important regulatory protein of microtubule dynamics, which can be directly targeted by Cdc2 [18]. In our analysis, we showed that stathmin ${ }^{\text {S38 }}$ phosphorylation was decreased (Figure 2, middle panels), which correlates with the inactivation of Cdc2 in N2a58/22L cells (Figure 2, right panels) implying that there is a functional interaction. Cdc2 is a crucial kinase in starting $M$ phase events during the cell cycle progression and regulates important mitotic structure changes, including nuclear envelope breakdown and spindle assembly [19]. Dephosphorylation of stathmin ${ }^{\text {S38 }}$ led to an inhibition of cells at G2/M phase, lack of spindle assembly, and growth inhibition
$[20,21]$. Together with the finding that the prion gene is transcriptionally activated in the G1 phase in confluent and terminally differentiated cells [22], we assume that control of the cell cycle might be important in prion diseases.

Aberrant signal transduction pathways are implicated in many diseases. However, perturbations in phosphorylation-based signaling networks are typically studied in a hypothesis-driven approach. In this study, we performed the first global analysis of the phosphoproteome of scrapie-infected neuronal cells, since the knowledge of PrPdependent deregulation of the signalling network is poor. SILAC provides a powerful and accurate technique for relative proteome-wide quantification by massspectrometry. Its versatility has been demonstrated by a wide range of applications, especially for intracellular signal transduction pathways [23-25]. Since we applied SILAC for the quantitative detection of the phosphoproteome in scrapie-infected neuroblastoma cells, we found 105 different phosphoproteins. Among identified proteins, we validated the regulated phosphorylation of cofilin, stathmin and $\mathrm{Cdc} 2$ indicating that the identification of phosphoproteins in scrapie-infected neuronal cells by SILAC is reliable. Future work is necessary to determine whether the identified novel phosphoproteins are involved in prion diseases and if they probably represent sensitive and specific biomarkers for diagnosis or therapeutic intervention strategies.

\section{Methods}

\section{Cell culture}

N2a58/22L cells have been described previously [11] and were kindly provided by Prof. Schätzl (LMU, Munich). Cells were cultured in DMEM containing 10\% FCS and $4 \mathrm{mM} \mathrm{L}$-glutamine at $37^{\circ} \mathrm{C}$. Cells were treated with $5 \mu \mathrm{g} / \mathrm{ml}$ pentosan polysulfate (Cartrophen Vet, A. Albrecht GmbH + Co. KG, Germany) for two passages, resulting in a stable rescued cell line for more than 15 passages (N2a58\# cells). Cell lysates were prepared by scraping cells in lysis buffer containing $150 \mathrm{mM} \mathrm{NaCl}$, $0.5 \%$ Triton X-100, 0.5\% DOC, $50 \mathrm{mM}$ Tris pH 7.5, 1 $\mathrm{mM}$ Na-vanadate, $1 \mathrm{mM} \mathrm{Na}$-molybdate, $20 \mathrm{mM} \mathrm{NaF}$, $10 \mathrm{mM} \mathrm{NaPP}, 20 \mathrm{mM} \beta$-glycerophosphat, $1 \times$ protease inhibitor cocktail (Roche, Mannheim, Germany). For digestion with proteinase $\mathrm{K}(\mathrm{PK}) 80 \mu \mathrm{g}$ protein were treated with $20 \mu \mathrm{g} / \mathrm{ml} \mathrm{PK}$ for $30 \mathrm{~min}$ at $37^{\circ} \mathrm{C}$. PK digestion was stopped by addition of laemmli sample buffer and protein denaturation at $95^{\circ} \mathrm{C}$ for $7 \mathrm{~min}$.

\section{Colony assay}

The colony assay was performed as previously described with minor modifications [26]. In brief, cells were grown on glass cover slips to confluence using a 24 well plate. The cell layer was soaked in lysis buffer $(150 \mathrm{mM} \mathrm{NaCl}$, 
Table 1 Proteins exhibiting decreased phosphorylation in N2a58/22L cells

\begin{tabular}{|c|c|c|c|c|c|c|c|}
\hline No. & Uniprot & Protein Names & Ratio $^{a}$ & Pept $^{\text {b }}$ & $\begin{array}{l}\text { sequence } \\
\text { coverage } \\
{[\%]}\end{array}$ & PEP $^{c}$ & $\begin{array}{l}\text { Biological } \\
\text { Process }\end{array}$ \\
\hline 1 & P43276 & Histone H1.5 & 0.46237 & 1 & 13.9 & $5.61 \mathrm{E}-16$ & nucleosome assembly \\
\hline 2 & P30681 & High mobility group protein B2 & 0.48683 & 2 & 11 & $3.33 \mathrm{E}-02$ & genome maintenance; differentiation \\
\hline 3 & P11440 & Cell division control protein 2 homolog & 0.49428 & 5 & 7.7 & $3.03 \mathrm{E}-03$ & cell cycles; protein phosphorylation \\
\hline 4 & P97310 & DNA replication licensing factor MCM2 & 0.54657 & 1 & 2.4 & $1.65 \mathrm{E}-05$ & $\begin{array}{l}\text { cell cycle; nucleosome assembly; } \\
\text { transcription }\end{array}$ \\
\hline 5 & P43275 & Histone H1.1 & 0.56637 & 1 & 19.2 & $8.42 \mathrm{E}-04$ & nucleosome assembly \\
\hline 6 & P43274 & Histone H1.4 & 0.58367 & 4 & 24.2 & $1.04 \mathrm{E}-14$ & nucleosome assembly \\
\hline 7 & Q9Z2X1-1 & Heterogeneous nuclear ribonucleoprotein $\mathrm{F}$ & 0.60054 & 2 & 6.5 & $5.45 \mathrm{E}-22$ & RNA processing \\
\hline 8 & P70670 & $\begin{array}{l}\text { Nascent polypeptide-associated complex } \\
\text { subunit alpha, muscle-specific form }\end{array}$ & 0.60501 & 2 & 1.2 & $5.07 \mathrm{E}-11$ & protein transport; transcription \\
\hline 9 & P60843 & Eukaryotic initiation factor $4 \mathrm{~A}-\mathrm{I}$ & 0.65781 & 5 & 4.9 & $5.39 \mathrm{E}-03$ & translation \\
\hline 10 & P27659 & 605 ribosomal protein $\mathrm{L} 3$ & 0.65906 & 12 & 7.7 & $1.49 \mathrm{E}-03$ & translation \\
\hline 11 & P28656 & Nucleosome assembly protein 1-like 1 & 0.70087 & 1 & 7.2 & $1.16 \mathrm{E}-05$ & nucleosome assembly \\
\hline 12 & Q62167 & ATP-dependent RNA helicase DDX3X & 0.70816 & 3 & 3.3 & $3.12 \mathrm{E}-19$ & putative helicase activity \\
\hline 13 & P68040 & $\begin{array}{l}\text { Guanine nucleotide-binding protein subunit } \\
\text { beta-2-like1 }\end{array}$ & 0.71371 & 1 & 7.9 & $5.15 \mathrm{E}-08$ & unknown \\
\hline 14 & P47911 & 605 ribosomal protein $\mathrm{L} 6$ & 0.71887 & 7 & 12 & $7.26 \mathrm{E}-07$ & translation \\
\hline 15 & Q61937 & Nucleophosmin & 0.72062 & 7 & 29.8 & $4.38 \mathrm{E}-07$ & cell cycle; nuclear export \\
\hline$\overline{16}$ & P15532 & Nucleoside diphosphate kinase $\mathrm{A}$ & 0.72108 & 2 & 17.8 & $7.93 \mathrm{E}-03$ & $\begin{array}{l}\text { NTP biosynthesis; nervous system } \\
\text { development }\end{array}$ \\
\hline 17 & O70251 & Elongation factor 1-beta & 0.73137 & 1 & 24 & $2.20 \mathrm{E}-18$ & translation \\
\hline 18 & Q61656 & Probable ATP-dependent RNA helicase DDX5 & 0.73832 & 4 & 3.7 & $1.41 \mathrm{E}-03$ & RNA processing; transcription \\
\hline 19 & Q9ERK4 & Exportin-2 & 0.74333 & 1 & 2.1 & $8.97 \mathrm{E}-05$ & cell proliferation; protein transport \\
\hline 20 & P09411 & Phosphoglycerate kinase 1 & 0.74902 & 2 & 6.7 & $3.40 \mathrm{E}-03$ & glycolysis; phosphorylation \\
\hline 21 & P48962 & ADP/ATP translocase 1 & 0.75149 & 6 & 18.8 & $3.87 \mathrm{E}-36$ & transmembrane transport \\
\hline 22 & Q9D8N0 & Elongation factor 1-gamma & 0.76191 & 3 & 7.8 & $1.45 \mathrm{E}-10$ & translation \\
\hline 23 & P49312-2 & Heterogeneous nuclear ribonucleoprotein A1 & 0.77303 & 5 & 12.1 & $7.20 \mathrm{E}-05$ & alternative splicing; nuclear export/import \\
\hline 24 & Q9CZM2 & $60 S$ ribosomal protein L15 & 0.77591 & 9 & 10.3 & $2.04 \mathrm{E}-15$ & translation \\
\hline 25 & P97855 & $\begin{array}{l}\text { Ras GTPase-activating protein-binding } \\
\text { protein } 1\end{array}$ & 0.78021 & 2 & 7.3 & $1.11 \mathrm{E}-15$ & protein transport \\
\hline 26 & Q9EQU5-1 & Protein SET & 0.79357 & 7 & 7.9 & $5.77 \mathrm{E}-12$ & nucleosome assembly \\
\hline 27 & Q7TPV4 & Myb-binding protein $1 \mathrm{~A}$ & 0.79496 & 2 & 1.5 & $2.39 \mathrm{E}-11$ & cytoplasmic transport; transcription \\
\hline 28 & P80318 & T-complex protein 1 subunit gamma & 0.79504 & 1 & 6.6 & $2.89 \mathrm{E}-05$ & protein folding \\
\hline 29 & P25444 & 40 S ribosomal protein S2 & 0.79516 & 20 & 12.3 & $2.99 \mathrm{E}-04$ & translation \\
\hline 30 & P10126 & Elongation factor 1-alpha 1 & 0.80203 & 5 & 18.6 & $1.06 \mathrm{E}-33$ & translational elongation \\
\hline 31 & P61979-2 & Heterogeneous nuclear ribonucleoprotein $\mathrm{K}$ & 0.80502 & 6 & 11.9 & $1.17 \mathrm{E}-15$ & RNA processing \\
\hline 32 & P07901 & Heat shock protein HSP 90-alpha & 0.80637 & 2 & 32.7 & $1.38 \mathrm{E}-94$ & $\begin{array}{l}\text { CD8 T-cell differentiation; chaperone } \\
\text { activity }\end{array}$ \\
\hline 33 & Q61598-1 & Rab GDP dissociation inhibitor beta & 0.81242 & 3 & 5.6 & $1.93 \mathrm{E}-05$ & $\begin{array}{l}\text { protein transport; regulation of GTPase } \\
\text { activity }\end{array}$ \\
\hline 34 & P54775 & $26 \mathrm{~S}$ protease regulatory subunit $6 \mathrm{~B}$ & 0.81795 & 2 & 7.2 & $1.59 \mathrm{E}-09$ & blastocyst development; protein catabolism \\
\hline 35 & Q20BD0 & Heterogeneous nuclear ribonucleoprotein A/B & 0.82349 & 3 & 19.3 & $3.26 \mathrm{E}-11$ & nucleotide binding \\
\hline 36 & P14206 & $40 \mathrm{~S}$ ribosomal protein SA;Laminin receptor 1 & 0.8261 & 7 & 20.7 & $2.57 \mathrm{E}-17$ & translation \\
\hline 37 & P68134 & Actin, alpha skeletal muscle & 0.83457 & 10 & 27.3 & $4.38 \mathrm{E}-26$ & cytoskeleton \\
\hline 38 & P80314 & T-complex protein 1 subunit beta & 0.83579 & 2 & 11.6 & $1.05 \mathrm{E}-21$ & protein folding \\
\hline 39 & P50580 & Proliferation-associated protein 2G4 & 0.84048 & 2 & 8.1 & $1.53 \mathrm{E}-03$ & rRNA processing; transcription; translation \\
\hline 40 & P11983-1 & T-complex protein 1 subunit alpha $B$ & 0.84687 & 4 & 8.8 & $5.73 \mathrm{E}-23$ & protein folding \\
\hline
\end{tabular}


Table 1: Proteins exhibiting decreased phosphorylation in N2a58/22L cells (Continued)

\begin{tabular}{|c|c|c|c|c|c|c|c|}
\hline 41 & P35564 & Calnexin & 0.85134 & 1 & 6.3 & $2.08 \mathrm{E}-06$ & protein folding \\
\hline 42 & Q8BUP7 & $\begin{array}{l}\text { Putative uncharacterized protein; } 26 \mathrm{~S} \text { protease } \\
\text { regulatory subunit } 6 \mathrm{~A}\end{array}$ & 0.85207 & 3 & 7.3 & $9.72 \mathrm{E}-03$ & blastocyst development; protein catabolism \\
\hline 43 & P63017 & Heat shock cognate $71 \mathrm{kDa}$ protein & 0.85947 & 9 & 32.2 & $2.16 \mathrm{E}-96$ & response to stress \\
\hline 44 & Q01768 & Nucleoside diphosphate kinase $B$ & 0.86107 & 4 & 17.8 & $1.35 \mathrm{E}-04$ & NTP biosynthesis \\
\hline 45 & P62082 & $40 S$ ribosomal protein $\mathrm{S7}$ & 0.86306 & 3 & 10.3 & $8.39 \mathrm{E}-03$ & translation \\
\hline 46 & P80315 & T-complex protein 1 subunit delta & 0.86423 & 1 & 6.9 & $3.81 \mathrm{E}-12$ & protein folding \\
\hline 47 & Q71LX8 & Heat shock protein $84 b$ & 0.8654 & 4 & 32 & $\begin{array}{l}6.13 \mathrm{E}- \\
136 \\
\end{array}$ & protein folding; stress response \\
\hline 48 & P58252 & Elongation factor 2 & 0.86672 & 2 & 7.9 & $3.57 \mathrm{E}-25$ & translation \\
\hline 49 & P08249 & Malate dehydrogenase, mitochondrial & 0.86743 & 1 & 9.2 & $2.49 \mathrm{E}-35$ & glycolysis \\
\hline 50 & P70168 & Importin subunit beta-1 & 0.87257 & 2 & 2.7 & $3.47 \mathrm{E}-13$ & nuclear import \\
\hline 51 & P51859 & Hepatoma-derived growth factor & 0.87395 & 2 & 12.7 & $2.95 \mathrm{E}-07$ & transcription \\
\hline 52 & P14152 & Malate dehydrogenase, cytoplasmic & 0.87522 & 1 & 7.8 & 1.09E-03 & glycolysis \\
\hline 53 & P80313 & T-complex protein 1 subunit eta & 0.89251 & 1 & 8.6 & $1.22 \mathrm{E}-39$ & protein folding \\
\hline 54 & P62827 & GTP-binding nuclear protein Ran & 0.89794 & 3 & 22.7 & 2.27E-09 & $\begin{array}{l}\text { cell cycle; nuclear import; signal } \\
\text { transduction }\end{array}$ \\
\hline 55 & P20029 & 78 kDa glucose-regulated protein & 0.89996 & 1 & 7 & $4.16 \mathrm{E}-04$ & $\begin{array}{l}\text { cerebellar Purkinje cell development/ } \\
\text { organization }\end{array}$ \\
\hline 56 & P56480 & ATP synthase subunit beta, mitochondrial & 0.90399 & 1 & 18.5 & $1.85 \mathrm{E}-42$ & proton transport; lipid metabolism \\
\hline 57 & P17742 & Peptidyl-prolyl cis-trans isomerase A & 0.90719 & 10 & 20.4 & 4.97E-20 & neuron differentiation; protein folding \\
\hline 58 & P20152 & Vimentin & 0.90814 & 10 & 26.2 & $2.22 \mathrm{E}-34$ & cytoskeleton \\
\hline 59 & P09103 & Protein disulfide-isomerase & 0.91019 & 2 & 4.7 & $8.84 \mathrm{E}-02$ & redox homeostasis \\
\hline 60 & P80317 & T-complex protein 1 subunit zeta & 0.91579 & 1 & 10.2 & $1.49 \mathrm{E}-02$ & protein folding \\
\hline 61 & Q569Z6 & $\begin{array}{l}\text { Thyroid hormone receptor-associated protein } \\
3\end{array}$ & 0.93507 & 3 & 3.7 & 4.27E-04 & transcription \\
\hline 62 & P09405 & Nucleolin & 0.93545 & 1 & 12.7 & $8.06 \mathrm{E}-34$ & nucleotide binding \\
\hline 63 & Q9D6F9 & Tubulin beta-4 chain & 0.93722 & 4 & 23.2 & $9.43 \mathrm{E}-32$ & cytoskeleton \\
\hline 64 & Q8C2Q7 & Heterogeneous nuclear ribonucleoprotein $\mathrm{H} 1$ & 0.93795 & 3 & 5.7 & $2.30 \mathrm{E}-06$ & nucleotide binding \\
\hline 65 & Q8K019-1 & Bcl-2-associated transcription factor 1 & 0.94132 & 4 & 5.3 & $2.22 \mathrm{E}-05$ & transcription \\
\hline 66 & P62908 & 40 S ribosomal protein $\mathrm{S3}$ & 0.94247 & 2 & 9.1 & $1.74 \mathrm{E}-06$ & translation \\
\hline 67 & P99024 & Tubulin beta- 5 chain & 0.9446 & 3 & 36.3 & 1.15E-54 & cytoskeleton \\
\hline 68 & P15331-2 & Peripherin & 0.95087 & 3 & 17.2 & $3.41 \mathrm{E}-40$ & cytoskeleton \\
\hline 69 & P63038-1 & $60 \mathrm{kDa}$ heat shock protein, mitochondrial & 0.96089 & 6 & 13.8 & $9.17 \mathrm{E}-37$ & T cell activation; interferon production \\
\hline 70 & P27773 & Protein disulfide-isomerase A3 & 0.96765 & 1 & 7.9 & $1.26 \mathrm{E}-03$ & redox homeostasis; apoptosis \\
\hline 71 & P16858 & Glyceraldehyde-3-phosphate dehydrogenase & 0.96943 & 58 & 23.4 & $5.69 \mathrm{E}-66$ & glycolysis \\
\hline 72 & Q3TED3 & $\begin{array}{l}\text { Putative uncharacterized protein; ATP-citrate } \\
\text { synthase }\end{array}$ & 0.97174 & 2 & 4.3 & $4.63 \mathrm{E}-03$ & acetyl-CoA biosynthesis \\
\hline 73 & Q03265 & ATP synthase subunit alpha, mitochondrial & 0.98925 & 2 & 13.6 & $1.81 \mathrm{E}-22$ & proton transport; lipid metabolism \\
\hline 74 & Q9ERD7 & Tubulin beta-3 chain & 0.99071 & 2 & 27.3 & $5.05 E-33$ & cytoskeleton \\
\hline 75 & P17751 & Triosephosphate isomerase & 0.9967 & 1 & 11.2 & $1.52 \mathrm{E}-04$ & gluconeogenesis; glycolysis \\
\hline
\end{tabular}

a. Ratio of N2a58/22L vs. N2a58\# cells

b. Number of identified peptides

c. posterior error probability (PEP) estimates the probability of wrong assignment of a spectrum to a peptide sequence

$0.5 \%$ Triton X-100, $0.5 \%$ DOC, $50 \mathrm{mM}$ Tris $\mathrm{pH} 7.5$ ) on a nitrocellulose membrane. After drying for $30 \mathrm{~min}$ at room temperature, the membrane was incubated in lysis buffer containing $5 \mu \mathrm{g} / \mathrm{ml}$ proteinase $\mathrm{K}$ (PK) for $90 \mathrm{~min}$ at $37^{\circ} \mathrm{C}$, rinsed twice with water, and incubated in $2 \mathrm{mM}$
PMSF for $10 \mathrm{~min}$. The membrane was shaken in $3 \mathrm{M}$ guanidinium thiocyanate, $10 \mathrm{mM}$ Tris- $\mathrm{HCl}(\mathrm{pH} \mathrm{8.0)}$ for $10 \mathrm{~min}$, followed by rinsing five times with water. $5 \%$ nonfat dry milk in TBS-T was used for blocking for $1 \mathrm{~h}$ at room temperature. PrP was detected using an anti-PrP 
Table 2 Proteins exhibiting increased phosphorylation in N2a58/22L cells

\begin{tabular}{|c|c|c|c|c|c|c|c|}
\hline No. & Uniprot & Protein Names & Ratio $^{a}$ & Pept. $^{\text {b }}$ & $\begin{array}{l}\text { Sequence } \\
\text { Coverage } \\
{[\%]}\end{array}$ & PEP $^{c}$ & $\begin{array}{l}\text { Biological } \\
\text { Process }\end{array}$ \\
\hline 76 & P80316 & T-complex protein 1 subunit epsilon & 1.017 & 1 & 6.1 & $8.63 \mathrm{E}-18$ & protein folding \\
\hline 77 & Q9CX22 & $\begin{array}{l}\text { Putative uncharacterized protein; } \\
\text { Cofilin-1 }\end{array}$ & 1.0173 & 5 & 29.7 & $3.72 \mathrm{E}-41$ & cytoskeleton; protein phosphorylation \\
\hline 78 & P32067 & Lupus La protein homolog & 1.02 & 2 & 7.5 & $1.68 \mathrm{E}-02$ & RNA processing \\
\hline 79 & A6ZI44 & Fructose-bisphosphate aldolase & 1.0328 & 8 & 12.2 & $2.75 \mathrm{E}-25$ & glycolysis \\
\hline 80 & P35700 & Peroxiredoxin-1 & 1.0342 & 6 & 6.7 & $2.44 \mathrm{E}-10$ & proliferation; redox homeostasis; stress response \\
\hline 81 & P05202 & $\begin{array}{l}\text { Aspartate aminotransferase, } \\
\text { mitochondrial }\end{array}$ & 1.0344 & 1 & 12.3 & $1.04 \mathrm{E}-11$ & aspartate biosynthesis; oxaloacetate metabolism \\
\hline 82 & Q3TFD0 & Serine hydroxymethyltransferase & 1.0425 & 3 & 6.5 & 2.95E-03 & carbon metabolism \\
\hline 83 & P63101 & 14-3-3 protein zeta/delta & 1.0478 & 19 & 19.2 & $7.09 \mathrm{E}-27$ & protein binding \\
\hline 84 & Q9CZ30-1 & Obg-like ATPase 1 & 1.0606 & 3 & 7.1 & $2.25 \mathrm{E}-16$ & ATP/GTP binding; hydrolase activity \\
\hline 85 & P06745 & Glucose-6-phosphate isomerase & 1.0652 & 3 & 7.7 & $3.34 \mathrm{E}-15$ & angiogenesis; gluconeogenesis; glycolysis \\
\hline 86 & Q71H74 & $\begin{array}{l}\text { Collapsin response mediator protein } \\
4 \mathrm{~A}\end{array}$ & 1.0688 & 5 & 6.3 & $6.94 \mathrm{E}-12$ & nervous system development \\
\hline 87 & Q6P5F9 & Exportin-1 & 1.0703 & 2 & 3.6 & $2.74 \mathrm{E}-20$ & nuclear export; centrosome duplication \\
\hline 88 & Q01853 & $\begin{array}{l}\text { Transitional endoplasmic reticulum } \\
\text { ATPase }\end{array}$ & 1.0708 & 2 & 3.7 & 2.97E-05 & apoptosis; retrograde protein transport \\
\hline 89 & Q3TCI7 & L-lactate dehydrogenase & 1.0746 & 4 & 18.3 & $1.43 \mathrm{E}-32$ & glycolysis \\
\hline 90 & P08113 & Endoplasmin & 1.08 & 1 & 2.6 & $1.05 \mathrm{E}-05$ & protein folding \\
\hline 91 & Q8VC46 & Ubc protein;Ubiquitin & 1.0848 & 17 & 30.7 & $2.18 \mathrm{E}-06$ & protein binding \\
\hline 92 & A0PJ96 & Mtap1b protein & 1.0871 & 2 & 3.2 & $2.72 \mathrm{E}-13$ & cytoskeleton \\
\hline 93 & Q61171 & Peroxiredoxin-2 & 1.0934 & 2 & 9.1 & 4.16E-04 & signal transduction; redox homeostasis \\
\hline 94 & Q9WVA4 & Transgelin-2 & 1.106 & 2 & 11.8 & 9.37E-05 & muscle organ development \\
\hline 95 & P52480-1 & Pyruvate kinase isozymes M1/M2 & 1.1081 & 3 & 22.2 & $6.30 \mathrm{E}-39$ & glycolysis \\
\hline 96 & 008709 & Peroxiredoxin-6 & 1.1204 & 5 & 13.8 & 1.10E-04 & redox homeostasis \\
\hline 97 & P17182 & Alpha-enolase & 1.128 & 11 & 41.5 & $6.35 \mathrm{E}-26$ & glycolysis \\
\hline 98 & P54227 & Stathmin & 1.1422 & 4 & 18.1 & $1.94 \mathrm{E}-02$ & cell cycle; cytoskeleton \\
\hline 99 & Q922F4 & Tubulin beta- 6 chain & 1.1427 & 1 & 19.2 & $1.01 \mathrm{E}-25$ & cytoskeleton \\
\hline 100 & Q60864 & Stress-induced-phosphoprotein 1 & 1.1499 & 1 & 3.5 & $5.12 \mathrm{E}-05$ & stress response \\
\hline 101 & P52480-2 & Pyruvate kinase isozymes M1/M2 & 1.2958 & 1 & 19.6 & $3.40 \mathrm{E}-33$ & glycolysis \\
\hline 102 & Q920E5 & Farnesyl pyrophosphate synthetase & 1.3534 & 1 & 8.2 & $8.44 \mathrm{E}-16$ & cholesterol/isoprenoid biosynthesis \\
\hline 103 & P38647 & Stress-70 protein, mitochondrial & 1.357 & 2 & 4.6 & 6.57E-08 & nuclear export; protein folding \\
\hline 104 & P14824 & Annexin A6 & 1.6252 & 4 & 7.9 & 2.47E-08 & $\mathrm{Ca}^{2+}$ transport; muscle contraction \\
\hline 105 & Q61753 & D-3-phosphoglycerate dehydrogenase & 1.7927 & 3 & 8.1 & $6.28 \mathrm{E}-15$ & cell cycle; neural development; serine biosynthesis \\
\hline
\end{tabular}

a. Ratio of N2a58/22L vs. N2a58\# cells

b. Number of identified peptides

c. posterior error probability (PEP) estimates the probability of wrong assignment of a spectrum to a peptide sequence

antibody 6H4 (Prionics) and a HRP-conjugated sheep anti-mouse antibody (GE Healthcare).

\section{SDS-PAGE and Western Blot}

Proteins were separated by $12 \%$ SDS-PAGE and transferred to polyvinylidene difluoride membranes (PVDF, Millipore) by semidry blotting. PrP was detected using the PrP-specific mouse mAb 8H4 (Alicon AG). For validation of phosphorylated proteins anti-phospho-stathmin (Ser38) (\#3426, Cell Signaling Technology), anti-phospho-cdc2 (Tyr15) (\#4539, Cell Signaling Technology), and anti-phospho-cofilin (Ser3) antibodies (\#3313, Cell Signaling Technology) were used. Antibodies recognizing
Table 3 Identified phosphorylation sites

\begin{tabular}{llll}
\hline Protein names & $\begin{array}{l}\text { Ratio } \\
\text { (total) }^{\mathbf{a}}\end{array}$ & Phosphosite & $\begin{array}{l}\text { Ratio } \\
\text { (specific phospho-site) }^{\mathbf{b}}\end{array}$ \\
\hline Cdc2 & 0.49428 & Y15 & 0.43086 \\
& & Y160 & 0.64359 \\
\hline stathmin & 1.1422 & S25 & 1.2155 \\
& & S38 & 0.45601 \\
\hline cofilin & 1.0173 & S3 & 1.6328 \\
\hline
\end{tabular}

a. Ratio of phosphorylation N2a58/22L vs. N2a58\# cells

b. Ratio of phospho sites in N2a58/22L and N2a58\# cells 

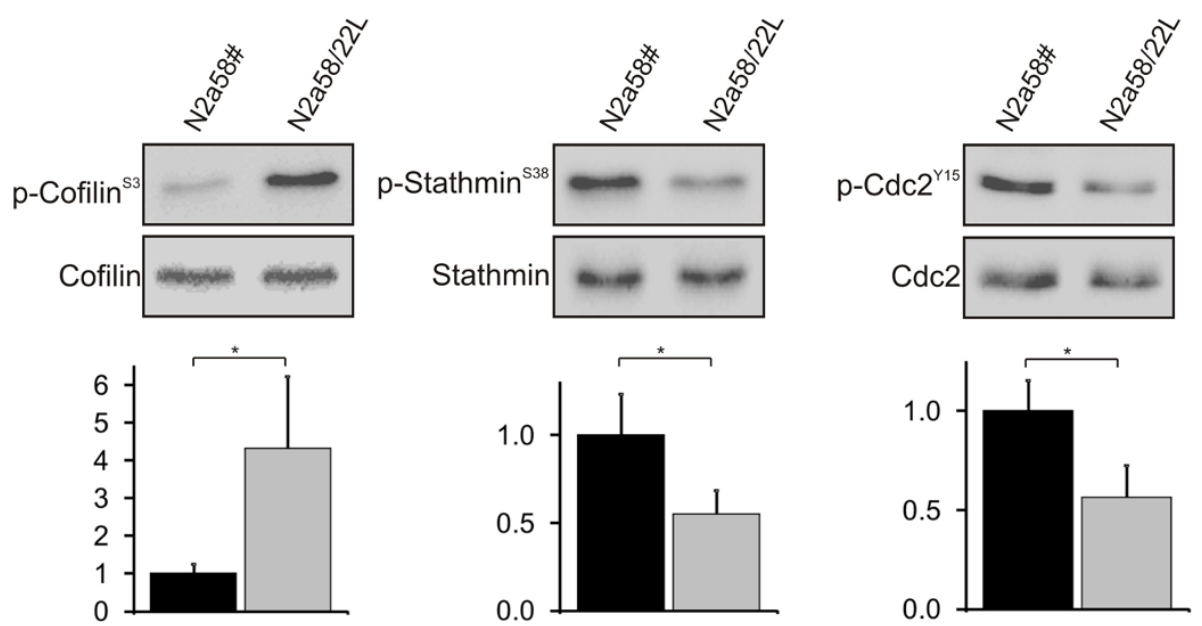

Figure 2 Specific regulation of cofilin, Cdc2, and stathmin phosphorylation in scrapie-infected neuronal cells. Cell lysates of N2a58\# and 22L-infected N2a58/22L cells were analyzed by Western blot using phospho-specific antibodies to detect p-cofilin ${ }^{\mathrm{S3}}, \mathrm{p}$-cdc2 $2^{\mathrm{Y} 15}$, and p-stathmin ${ }^{\mathrm{S38}}$ (left panels). As loading controls, equal amounts of cofilin, Cdc2 and stathmin were shown. Quantification of intensities of phosphorylation signal was performed by normalizing the corresponding loading control $\left.{ }^{*} p<0.05\right)$ (right panels).

stathmin (\#3352), cdc2 (\#9112) and cofilin (\#3312) were also obtained from Cell Signaling Technology.

\section{Two dimensional gel electrophoresis}

For 2D electrophoresis $150 \mu \mathrm{g}$ protein of cell lysates were purified by trichloroacetic acid precipitation and re-suspended in DeStreak Rehydration Solution (Amersham Biosciences) containing 0.5\% Bio-Lyte pH3-10 (Bio-Rad Laboratories $\mathrm{GmbH}$, München). The isoelectric focusing was run on IPG strips with a non-linear $\mathrm{pH}$ range of 3-10 and a length of $7 \mathrm{~cm}$ (Bio-Rad) using the $\mathrm{ZOOM}^{\circ}$ IPGRunner ${ }^{\text {Tw }}$ system from Invitrogen. After focussing strips were equilibrated in $50 \mathrm{mM}$ Tris, $1 \mathrm{mM}$ Urea, 30\% Glycerin, 2\% SDS, 1\% DTT for $25 \mathrm{~min}$ and in $50 \mathrm{mM}$ Tris, $1 \mathrm{mM}$ Urea, 30\% Glycerin, 2\% SDS, 5\% Iodacetamid for $25 \mathrm{~min}$. Strips were then separated in 10\% SDS-PAGE gels in the second dimension and analyzed by Coomassie staining or immunoblotting using an anti-phospho-tyrosine (sc-7020, Santa Cruz) or an anti-phospho-threonine antibody (\#9381, Cell Signaling Technology).

\section{SILAC phosphoproteomics analysis}

SILAC ready-to-use cell culture media and dialyzed FBS were obtained from Dundee Cell Products Ltd, UK. While N2a58\# cells were cultured in control SILAC DMEM media containing unlabelled arginine and lysine amino acids (R0K0), N2a58/22L cells were cultured in ready-to-use SILAC DMEM medium containing ${ }^{13} \mathrm{C}$ labeled arginine and lysine amino acids (R6K6) for seven cell division cycles. After preparation of cell lysates and measurement of protein concentration, lysates of N2a58\# and N2a58/22L cells were mixed in a ratio 1:1. Each sample was reduced in SDS PAGE loading buffer containing $10 \mathrm{mM}$ DTT and alkylated in $50 \mathrm{mM}$ iodoacetamide prior to separation by one-dimensional SDSPAGE (4-12\% Bis-Tris Novex mini-gel, Invitrogen) and visualization by colloidal Coomassie staining (Novex, Invitrogen). The entire protein gel lane was excised and cut into 10 gel slices each. Every gel slice was subjected to in-gel digestion with trypsin [27]. The resulting tryptic peptides were extracted by $1 \%$ formic acid, acetonitrile, lyophilized in a speedvac (Helena Biosciences).

\section{Phosphopeptide enrichment}

The lyophilized peptides above were resuspended in 5\% acetic acid (binding buffer) and phosphopeptide enrichment was carried out using immobilized metal ion affinity chromatography (IMAC). Immobilized gallium in the Pierce Ga-IDA Phosphopeptide Enrichment Kit was used to enrich for phosphopeptides prior to MS/MS analysis according to the manufacturer's instructions (Thermo Scientific).

\section{LC-MS/MS}

Trypsin digested peptides were separated using an Ultimate U3000 (Dionex Corporation) nanoflow LC-system consisting of a solvent degasser, micro and nanoflow pumps, flow control module, UV detector and a thermostated autosampler. $10 \mu \mathrm{l}$ of sample (a total of $2 \mu \mathrm{g}$ ) was loaded with a constant flow of $20 \mu \mathrm{l} / \mathrm{min}$ onto a PepMap C18 trap column $(0.3 \mathrm{~mm}$ id $\times 5 \mathrm{~mm}$, Dionex Corporation). After trap enrichment peptides were eluted off onto a PepMap C18 nano column $(75 \mu \mathrm{m} \times$ $15 \mathrm{~cm}$, Dionex Corporation) with a linear gradient of $5-35 \%$ solvent B ( $90 \%$ acetonitrile with $0.1 \%$ formic acid) over 65 minutes with a constant flow of $300 \mathrm{nl} / \mathrm{min}$. 
The HPLC system was coupled to a LTQ Orbitrap XL (Thermo Fisher Scientific Inc) via a nano ES ion source (Proxeon Biosystems). The spray voltage was set to $1.2 \mathrm{kV}$ and the temperature of the heated capillary was set to $200^{\circ} \mathrm{C}$. Full scan MS survey spectra (m/z 3351800 ) in profile mode were acquired in the Orbitrap with a resolution of 60,000 after accumulation of 500,000 ions. The five most intense peptide ions from the preview scan in the Orbitrap were fragmented by collision induced dissociation (normalised collision energy $35 \%$, activation Q 0.250 and activation time $30 \mathrm{~ms}$ ) in the LTQ after the accumulation of 10,000 ions. Maximal filling times were $1,000 \mathrm{~ms}$ for the full scans and $150 \mathrm{~ms}$ for the MS/MS scans. Precursor ion charge state screening was enabled and all unassigned charge states as well as singly charged species were rejected. The dynamic exclusion list was restricted to a maximum of 500 entries with a maximum retention period of 90 seconds and a relative mass window of $10 \mathrm{ppm}$. The lock mass option was enabled for survey scans to improve mass accuracy [28]. Data were acquired using the Xcalibur software.

\section{Quantification and Bioinformatic Analysis}

Quantification was performed with MaxQuant version 1.0.7.4 [29], and was based on two-dimensional centroid of the isotope clusters within each SILAC pair. To minimize the effect of outliers, protein ratios were calculated as the median of all SILAC pair ratios that belonged to peptides contained in the protein. The percentage variability of the quantitation was defined as the standard deviation of the natural logarithm of all ratios used for obtaining the protein ratio multiplied by a constant factor 100 .

The generation of peak list, SILAC- and extracted ion current-based quantitation, calculated posterior error probability, and false discovery rate based on search engine results, peptide to protein group assembly, and data filtration and presentation was carried out using MaxQuant. The derived peak list was searched with the Mascot search engine (version 2.1.04; Matrix Science, London, UK) against a concatenated database combining 80,412 proteins from International Protein Index (IPI) human protein database version 3.6 (forward database), and the reversed sequences of all proteins (reverse database). Alternatively, database searches were done using Mascot (Matrix Science) as the database search engine and the results saved as a peptide summary before quantification using MSQuant http://msquant.sourceforge. net/. Parameters allowed included up to three missed cleavages and three labeled amino acids (arginine and lysine). Initial mass deviation of precursor ion and fragment ions were up to $7 \mathrm{ppm}$ and $0.5 \mathrm{Da}$, respectively.
The minimum required peptide length was set to 6 amino acids. To pass statistical evaluation, posterior error probability (PEP) for peptide identification (MS/MS spectra) should be below or equal to 0.1 . The required false positive rate (FPR) was set to $5 \%$ at the peptide level. False positive rates or PEP for peptides were calculated by recording the Mascot score and peptide sequence length-dependent histograms of forward and reverse hits separately and then using Bayes' theorem in deriving the probability of a false identification for a given top scoring peptide. At the protein level, the false discovery rate (FDR) was calculated as the product of the PEP of a protein's peptides where only peptides with distinct sequences were taken into account. If a group of identified peptide sequences belong to multiple proteins and these proteins cannot be distinguished, with no unique peptide reported, these proteins are reported as a protein group in MaxQuant. Proteins were quantified if at least one MaxQuant-quantifiable SILAC pair was present. Identification was set to a false discovery rate of $1 \%$ with a minimum of two quantifiable peptides. The set value for FPR/PEP at the peptide level ensures that the worst identified peptide has a probability of 0.05 of being false; and proteins are sorted by the product of the false positive rates of their peptides where only peptides with distinct sequences are recognized. During the search, proteins are successively included starting with the bestidentified ones until a false discovery rate of $1 \%$ is reached; an estimation based on the fraction of reverse protein hits.

Enzyme specificity was set to trypsin allowing for cleavage of $\mathrm{N}$-terminal to proline and between aspartic acid and proline. Carbamidomethylation of cysteine was searched as a fixed modification, whereas $\mathrm{N}$-acetyl protein, oxidation of methionine and phosphorylation of serine, threonine and tyrosine were searched as variable modifications.

\section{Acknowledgements}

We thank Prof. Schätzl from the LMU in Munich for providing N2a58/22L cells.

\section{Author details}

${ }^{1}$ Paul Ehrlich Institute, Paul Ehrlich-Straße 51-59, D-63225 Langen, Germany.

${ }^{2}$ Dundee Cell Products Ltd, James Lindsay Place, Dundee Technopole Dundee, DD1 5JJ, UK. ${ }^{3}$ Division of Microbiology, Paris-Lodron University, Salzburg, Austria.

\section{Authors' contributions}

WW carried out the experimental work, drafted and wrote the manuscript. PA performed and interpreted the SILAC analysis. $J$ participated in the design of the study. SW conceived of the study, and participated in its design and coordination and wrote the manuscript. All authors read and approved the final manuscript.

\section{Competing interests}

The authors declare that they have no competing interests. 
Received: 18 August 2010 Accepted: 28 September 2010

Published: 28 September 2010

\section{References}

1. Prusiner SB: Prions. Proc Natl Acad Sci USA 1998, 95:13363-13383.

2. Prusiner SB: Novel proteinaceous infectious particles cause scrapie. Science 1982, 216:136-144.

3. Aguzzi A, Polymenidou M: Mammalian prion biology: one century of evolving concepts. Cell 2004, 116:313-327.

4. Taylor DR, Hooper NM: The prion protein and lipid rafts. Mol Membr Biol 2006, 23:89-99.

5. Mattei V, Garofalo T, Misasi R, Circella A, Manganelli V, Lucania G, Pavan A, Sorice M: Prion protein is a component of the multimolecular signaling complex involved in T cell activation. FEBS Lett 2004, 560:14-18.

6. Hugel B, Martinez MC, Kunzelmann C, Blattler T, Aguzzi A, Freyssinet JM: Modulation of signal transduction through the cellular prion protein is linked to its incorporation in lipid rafts. Cell Mol Life Sci 2004, 61:2998-3007.

7. Mouillet-Richard S, Ermonval M, Chebassier C, Laplanche JL, Lehmann S, Launay JM, Kellermann O: Signal transduction through prion protein. Science 2000, 289:1925-1928.

8. Gyllberg H, Lofgren $\mathrm{K}$, Lindegren $\mathrm{H}$, Bedecs K: Increased Src kinase level results in increased protein tyrosine phosphorylation in scrapie-infected neuronal cell lines. FEBS Lett 2006, 580:2603-2608.

9. Na YJ, Jin JK, Kim JI, Choi EK, Carp RI, Kim YS: JAK-STAT signaling pathway mediates astrogliosis in brains of scrapie-infected mice. J Neurochem 2007, 103:637-649.

10. Ertmer A, Gilch S, Yun SW, Flechsig E, Klebl B, Stein-Gerlach M, Klein MA, Schatzl HM: The tyrosine kinase inhibitor STI571 induces cellular clearance of PrPSc in prion-infected cells. J Biol Chem 2004, 279:41918-41927.

11. Nishida N, Harris DA, Vilette D, Laude H, Frobert $Y$, Grassi J, Casanova D, Milhavet O, Lehmann S: Successful transmission of three mouse-adapted scrapie strains to murine neuroblastoma cell lines overexpressing wildtype mouse prion protein. J Virol 2000, 74:320-325.

12. Kocisko DA, Engel AL, Harbuck K, Arnold KM, Olsen EA, Raymond LD, Vilette D, Caughey B: Comparison of protease-resistant prion protein inhibitors in cell cultures infected with two strains of mouse and sheep scrapie. Neurosci Lett 2005, 388:106-111.

13. Kascsak RJ, Rubenstein R, Merz PA, Carp Rl, Wisniewski HM, Diringer H: Biochemical differences among scrapie-associated fibrils support the biological diversity of scrapie agents. J Gen Virol 1985, 66(Pt 8):1715-1722.

14. Collinge J, Sidle KC, Meads J, Ironside J, Hill AF: Molecular analysis of prion strain variation and the aetiology of 'new variant' CJD. Nature 1996, 383:685-690.

15. Somerville RA, Chong A, Mulqueen OU, Birkett CR, Wood SC, Hope J: Biochemical typing of scrapie strains. Nature 1997, 386:564.

16. Yang N, Higuchi O, Ohashi K, Nagata K, Wada A, Kangawa K, Nishida E, Mizuno K: Cofilin phosphorylation by LIM-kinase 1 and its role in Racmediated actin reorganization. Nature 1998, 393:809-812.

17. Giorgi A, Di Francesco L, Principe S, Mignogna G, Sennels L, Mancone C Alonzi T, Sbriccoli M, De Pascalis A, Rappsilber J, et al: Proteomic profiling of PrP27-30-enriched preparations extracted from the brain of hamsters with experimental scrapie. Proteomics 2009, 9:3802-3814.

18. Beretta L, Dobransky T, Sobel A: Multiple phosphorylation of stathmin. Identification of four sites phosphorylated in intact cells and in vitro by cyclic AMP-dependent protein kinase and p34cdc2. J Biol Chem 1993, 268:20076-20084.

19. Satyanarayana A, Kaldis P: Mammalian cell-cycle regulation: several Cdks, numerous cyclins and diverse compensatory mechanisms. Oncogene 2009, 28:2925-2939.

20. Brattsand G, Marklund U, Nylander K, Roos G, Gullberg M: Cell-cycleregulated phosphorylation of oncoprotein 18 on Ser16, Ser25 and Ser38. Eur J Biochem 1994, 220:359-368.

21. Marklund U, Osterman O, Melander H, Bergh A, Gullberg M: The phenotype of a "Cdc2 kinase target site-deficient" mutant of oncoprotein 18 reveals a role of this protein in cell cycle control. J Biol Chem 1994, 269:30626-30635.

22. Gougoumas DD, Vizirianakis IS, Tsiftsoglou AS: Transcriptional activation of prion protein gene in growth-arrested and differentiated mouse erythroleukemia and human neoplastic cells. Exp Cell Res 2001, 264:408-417.

23. Zhang G, Neubert TA: Use of stable isotope labeling by amino acids in cell culture (SILAC) for phosphotyrosine protein identification and quantitation. Methods Mol Biol 2009, 527:79-92, xi.

24. Amanchy R, Kalume DE, Iwahori A, Zhong J, Pandey A: Phosphoproteome analysis of HeLa cells using stable isotope labeling with amino acids in cell culture (SILAC). J Proteome Res 2005, 4:1661-1671.

25. Kruger M, Kratchmarova I, Blagoev B, Tseng YH, Kahn CR, Mann M: Dissection of the insulin signaling pathway via quantitative phosphoproteomics. Proc Natl Acad Sci USA 2008, 105:2451-2456.

26. Klohn PC, Stoltze L, Flechsig E, Enari M, Weissmann C: A quantitative, highly sensitive cell-based infectivity assay for mouse scrapie prions. Proc Natl Acad Sci USA 2003, 100:11666-11671

27. Shevchenko A, Wilm M, Vorm O, Mann M: Mass spectrometric sequencing of proteins silver-stained polyacrylamide gels. Anal Chem 1996, 68:850-858.

28. Olsen JV, de Godoy LM, Li G, Macek B, Mortensen P, Pesch R, Makarov A, Lange $\mathrm{O}$, Horning $\mathrm{S}$, Mann M: Parts per million mass accuracy on an Orbitrap mass spectrometer via lock mass injection into a C-trap. Mol Cell Proteomics 2005, 4:2010-2021.

29. Cox J, Mann M: MaxQuant enables high peptide identification rates, individualized p.p.b.-range mass accuracies and proteome-wide protein quantification. Nat Biotechnol 2008, 26:1367-1372.

doi:10.1186/1478-811X-8-28

Cite this article as: Wagner et al:: Quantitative phosphoproteomic analysis of prion-infected neuronal cells. Cell Communication and Signaling 2010 8:28.

\section{Submit your next manuscript to BioMed Central and take full advantage of:}

- Convenient online submission

- Thorough peer review

- No space constraints or color figure charges

- Immediate publication on acceptance

- Inclusion in PubMed, CAS, Scopus and Google Scholar

- Research which is freely available for redistribution
Ciomed Central 